\title{
Compression depth declines below CPR guidelines when rate exceeds guidelines
}

JK Russell M Leturiondo² DM Gonzalez-Otero ${ }^{2,3^{*}}$ J Ruiz² M Daya ${ }^{1}$ S Ruiz de Gauna ${ }^{2}$

*dignamaria.gonzalez@ehu.eus

1 Oregon Health \& Science University (OHSU), Portland OR, USA

2 University of the Basque Country, UPV/EHU, Bilbao, Spain

${ }^{3}$ Bexen Cardio, Ermua, Spain

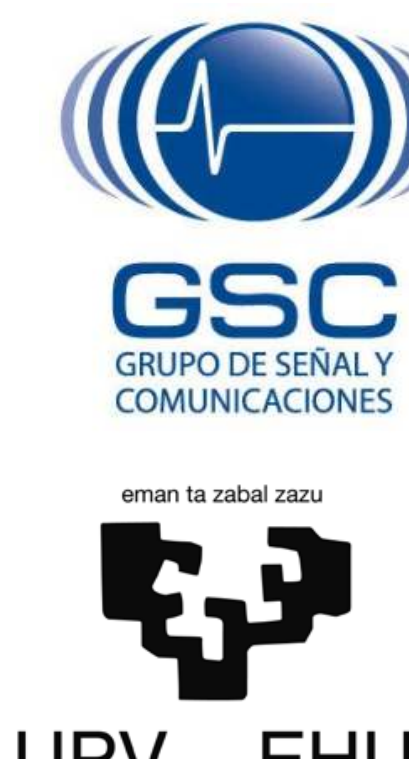

UPV EHU

\section{INTRODUCTION}

CPR guidelines recommend limits for rate and depth of chest compressions. Little is known about the interactions between rate and depth. We analyzed the relation between rate and depth of chest compressions during continuous chest compressions (CCC) CPR in out-of-hospital resuscitations.

Aim: To determine the dependency of chest compression depth on chest compression rate during CPR

\section{MATERIALS}

Rate and depth signals were extracted from adult cases of manual CPR during out-of-hospital resuscitations attended by a single EMS agency (TVFR, Tigard, Oregon USA) during 2013 through 2017.

Signals were extracted from monitor/defibrillators equipped with CPR monitors. TVFR performs CCC CPR. Rescuers received real-time feedback on rate, depth and leaning.

\section{RESULTS}

Cases $(n=616)$ included $1726(1046$ - 2609) (median, IQR) compressions (total: 1.172 .873 compressions). The model shows that, controlling for differences among subjects and position in the rescue, depth depends on rate:

$$
\text { Depth }_{m m}=64.4( \pm 0.36)-0.12( \pm 0.001){ }_{(p<0.001)}^{*} \text { Rate }_{(p p m}
$$

Observed values are shown in the figure. Above a rate of $90 \mathrm{cpm}$, compression depths are well modeled as declining linearly with rate. Above the upper recommended bound on rate of $120 \mathrm{cpm}$, median depth falls below recommended minimum of $50 \mathrm{~mm}$ and it continues to decline at higher rates.

\section{METHODS}

We computed compression depth and velocity from recorded acceleration using forward and reverse digital filtering to preserve phase. Chest compressions were automatically identified in the velocity signal using a threshold of $25 \mathrm{~mm} / \mathrm{s}$, and were required to have a peak force of at least $5 \mathrm{~kg}-f$. Additional rules excluded instances with highly irregular shapes. Peak depth and duration were measured for each compression. Rate was calculated as the inverse of duration.

The dependence of depth on rate was calculated with a generalized linear mixed effect model, treating differences between cases and position in the rescue as random effects.

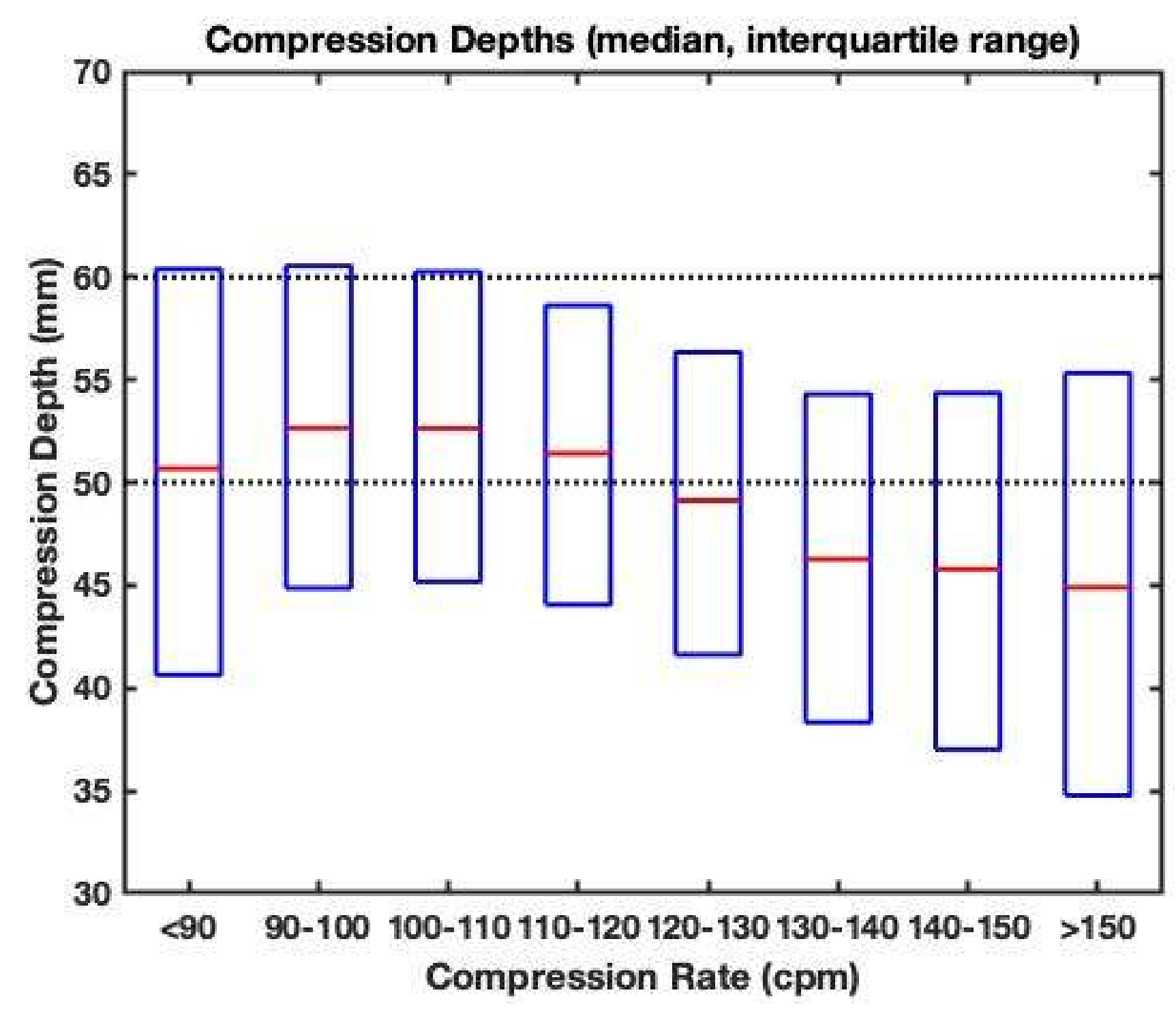

\section{CONCLUSIONS}

In out-of-hospital manual CPR, compression depth declines with compression rate. Median depth falls below guideline recommendation when rate exceeds current guideline recommendations during CCC CPR. 